\begin{tabular}{|c|c|c|l|l|}
\multirow{2}{*}{ SPi } & Journal Code & Article ID & Dispatch: 01-JUL-19 & CE: \\
\cline { 2 - 5 } & AIC & 16709 & No. of Pages: 7 & ME: \\
\hline
\end{tabular}

\title{
Ability of chromatographic mass balance to predict solute diffusivity in drug delivery systems
}

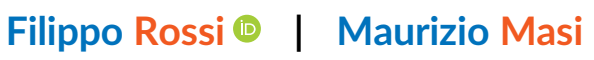

Department of Chemistry, Materials and Chemical Engineering "Giulio Natta",

Politecnico di Milano, Milan, Italy

\section{Correspondence}

Filippo Rossi, Department of Chemistry, Materials and Chemical Engineering "Giulio Natta", Politecnico di Milano, via Mancinelli 7, 20131 Milan, Italy.

Email: filippo.rossi@polimi.it

\begin{abstract}
The ability to predict the drug diffusion coefficient within hydrogel-based drug delivery devices has a pivotal role in the design of these materials. In the last years, many mathematical models have been developed, but they often rely on fitted parameters with a consequent limitation in terms of prediction. Indeed, they are mainly centered on the pure Fickian diffusion together with degradation and swelling contributions. However, especially with a drug concentration typical of pharmacological treatments, several other mechanisms such as drug-polymer and drug-drug interactions cannot be neglected. In this work, we checked the ability of a simple mathematical model to estimate diffusion coefficients of drugs loaded within hydrogel considered as a chromatographic stationary phase. Mathematical modeling satisfactorily matched with different sets of literature data proving that our assumptions are able to describe the key phenomena governing the device's behavior.

KEYWORDS

adsorption/liquid, chromatography, diffusion (in polymers), drug release, mathematical modeling
\end{abstract}

\section{1 | INTRODUCTION}

It is worldwide known that the possibility to control and sustain the release of drugs in the target tissue has represented a promising strategy for almost 50 years now. ${ }^{1-3}$ Briefly, following this strategy it is possible to avoid risks due to overdosing, together with the inefficacy of underdosing, in order to maintain drug levels within a therapeutic range with a consequent lower amount of drug needed. ${ }^{4-6}$ In addition, the smart possibility to control the release of molecules through a device reduces the risks linked to surgery or multiple treatments. ${ }^{7-9}$ Taking advantage of this technology, many products have been developed and are already on the market or used in clinical trials. ${ }^{2,10}$ Among such products, hydrogels hold great promises for many biomedical applications. Moreover, significant progress has been made in designing, synthesizing, and using these materials in different districts and diseases. ${ }^{11-15}$ Thanks to their high flexibility and biocompatibility, they represent an ideal hydrophilic three-dimensional network capable of carrying drugs, nanoparticles and cells. ${ }^{16-18}$ Hence, to improve the delivery performance of hydrogels, a deep understanding of the solute diffusion in gel matrices is pivotal. ${ }^{19,20}$ An examination of the state of the art provides both experimental studies and phenomenological theories related to the diffusion mechanism of molecules from hydrophilic macromolecular 3D networks. ${ }^{21-23}$ These theoretical descriptions can be divided into three main categories: (a) free-volume-based theories, where a solute diffusion in pure liquids was extended to polymeric systems; (b) hydrodynamic theories, that assumes the enhancement of frictional drag on the solute by slowing down the fluid flow in the proximity of polymeric chains; and (c) obstruction theories, where polymer chains are described as an almost impenetrable network that increases the effective path length of diffusive transport. ${ }^{24-26}$ Unfortunately these three theories are not able to cover all the possibilities, mainly due to the fact that they consider only the role of pure-Fickian diffusion within the 3D polymeric network. ${ }^{21,23}$

In the last years, many research groups have started to consider also other mechanisms just as important to predict mass diffusivities within hydrogels. ${ }^{26,27}$ 
Hadjiev and coworkers considered the ability of the obstructionscaling model to provide reasonable estimates of solute diffusion coefficients within hydrogels, as well as the assumption that a hydrogel can be represented as an entangled polymer solution of an equivalent concentration. $^{26}$ Liu and coworkers considered the importance of drugpolymer interactions described with local equilibrium and Henry's law. ${ }^{27}$ Kotsmar et al. quantified the mesh size and determined how gel matrices interact with solutes. ${ }^{28}$ Rossi and coworkers considered the parallelism between hydrogels for drug delivery and chromatography. For the first time they modeled the release of small steric hindrance drugs starting from chromatographic mass balance. ${ }^{29,30}$ The main difference, between these two disciplines that seem to be too far each other, resides in the fact that in drug delivery it is possible to neglect convection (assumption of hydrogels with small porosity and without forced flow conditions) and consider only diffusion in term of mass transport. ${ }^{31,32}$ Points in com1 mon are several and schematized in Figure 1. Indeed, in both systems hydrogel matrices can work as a stationary phase. They can be loaded with solute molecules diffused within the matrix interacting and adsorbing it (solute-polymer interaction) and interacting between them (solute-solute interactions). Accordingly, the aim of this study is to develop a mathematical model to predict drug diffusivity though hydrogels: such model should be simple with respect to numerical solution (thus avoiding computational expensive simulations, as it occurs with FEM); at the same time, though, it should be able to take into account all fundamental phenomena that influence the final behavior.

Several systems from literature were analyzed to prove model reliability and hypothesis coherence by comparing simulation results with experimental data. ${ }^{27,28,33-35}$ This represents the validation with litera- 54 ture data of the joint between chromatography and drug delivery that 55 could pave the way for a better design of these medical devices. 56

\section{2 | MODEL DEVELOPMENT}

This work presents a model for the prediction of solute diffusivity in the case of: (a) hydrogels with nanopores (convection neglectable); (b) low steric hindrance hydrophilic drugs typical of corticosteroids and anti-inflammatory drugs; (c) characteristic time of release mechanism shorter than the swelling and degradation of the polymeric network. Therefore, being the drug hydrodynamic radius smaller than the mean mesh size, drug molecules are not physically entrapped and so the motion should be driven only by the Fickian diffusion. The key role of adsorption (drug-polymer interactions) and drug aggregation (drug-drug interactions) proposed in previous work is here validated with literature data. In Figure 2, the two cases of drugs with notendency to aggregate (nonaggregative drugs) or to form dimers or trimers (aggregative drugs) are schematized.

\subsection{Mathematical model for nonaggregative drugs}

In Figure 2, the solid lines represent the hydrogel matrix, the black circles represent the drug molecules adsorbed onto the network backbone, and the red circles represent drug molecules free to move within the network. As mentioned in the introduction, to estimate

\section{CHROMATOGRAPHY}
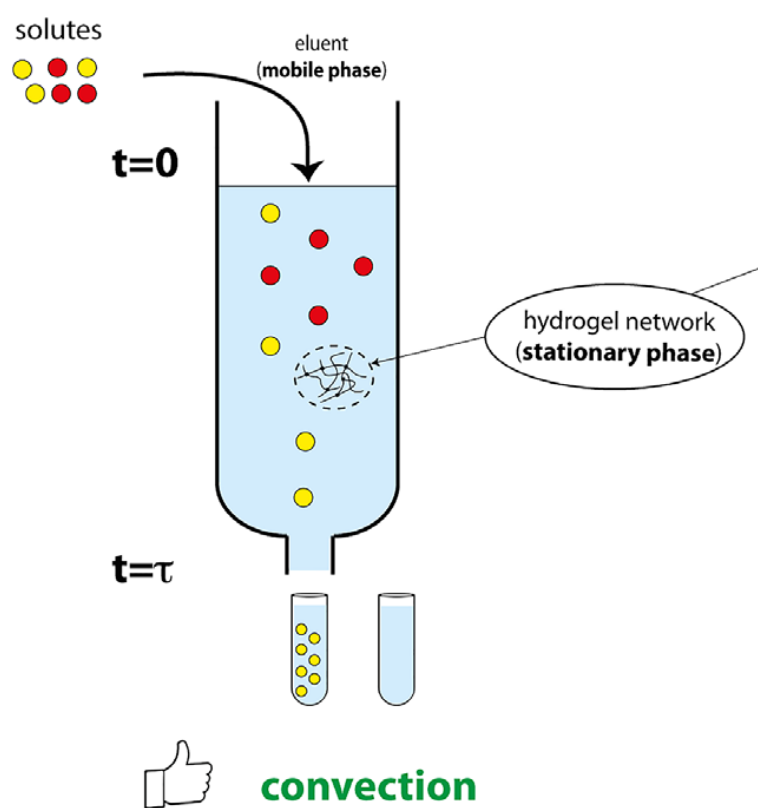

diffusion

\section{DRUG DELIVERY}

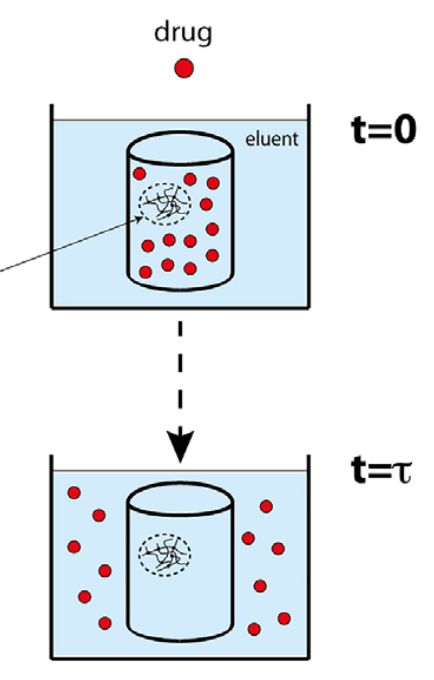

diffusion

convection
FIGURE 1 Schematic comparison between chromatography and drug delivery: stationary phase (hydrogels) and mobile phase (eluent) [Color figure can be viewed at wileyonlinelibrary.com] 
FIGURE 2 Pictorial representation of partitioning models in the case of drugs that can create oligomers (aggregative drug) or cannot (nonaggregative drug) [Color figure can be viewed at wileyonlinelibrary.com]

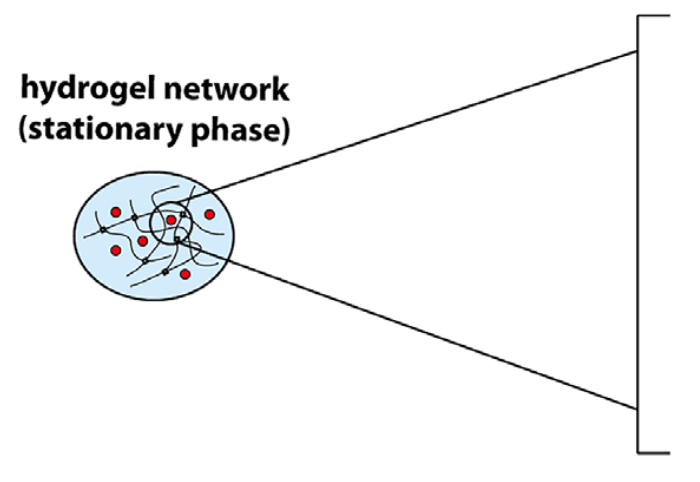

non aggregative drug

00000000000

$0_{>0 \rightarrow 0^{\pi}}$

$\bullet \bullet \bullet \bullet \bullet \bullet \bullet \bullet \bullet \bullet \bullet \bullet ~$

aggregative drug

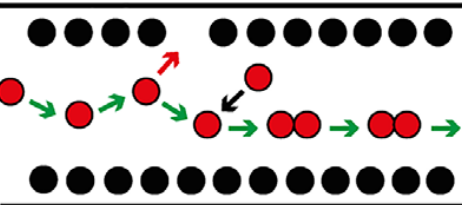

- drug adsorbed (q)

$\rightarrow$ collision (aggregation)

— hydrogel matrix

$\longrightarrow$ diffusion diffusivity this study started from the mass balance commonly used in chromatography:

$$
\varepsilon \cdot D_{\text {water }} \cdot \frac{\partial^{2} C_{G}}{\partial x^{2}}-u \cdot \frac{\partial C_{G}}{\partial x}=\varepsilon \cdot \frac{\partial C_{G}}{\partial t}+(1-\varepsilon) \cdot \frac{\partial q}{\partial t}
$$

where $\varepsilon$ is the gel porosity, $C_{\mathrm{G}}$ is the drug concentration within the hydrogel, $q$ is the drug adsorbed and $u$ is the superficial velocity of chromatographic columns, $D_{\text {water }}$ is the diffusivity of the specie in water environment. Generally, in chromatography the first term that takes into account the diffusion mechanism is neglected. Here, its contribution is considered, while the second one is not considered because, as mentioned, there is no presence of pressure that induces a flow rate typical of analytes.

Following mathematics published by our group ${ }^{30,36}$ we can obtain gel diffusivity $\left(D_{\text {gel }}\right)$ as:

$$
D_{\text {gel }}=\frac{\varepsilon \cdot D_{\text {water }}}{\left(\varepsilon+(1-\varepsilon) \cdot \frac{q^{\infty} \cdot K}{\Delta^{2}}\right)}
$$

where $D_{\text {gel }}$ is the diffusivity of the specie in gel environment and $\Delta=1+K \cdot C_{G}$

So the diffusivity of drug molecules in gel environment depends on:

1. motion in release environment ( $\left.D_{\text {water }}\right)$;

2. hydrogel structural property $(\varepsilon)$;

3. affinity between drug molecules and polymeric network $(K)$;

4. saturation of hydrogel adsorbing sites $(q \infty)$

5. drug concentration (contained in $\Delta$ ).

To understand the last contribution, that is counterintuitive, we should imagine that drug molecules are firstly interacting and adsorbed in the pores (red arrows in Figure 2) present within the gel until saturation of these adsorbing sites. Then, increasing drug concentration, no more adsorbing sites are available and transport occur by mass diffusion (green arrows in Figure 2) driven by concentration gradient present between the inner part of the hydrogel and the release environment.

\subsection{Mathematical model for aggregative drugs}

In previous works we hypothesized that the ability of drug molecules to aggregate in dimers and trimers influences their behavior in term of drug delivery from hydrogel and it is different respect to what is happening in aqueous solutions. ${ }^{37}$ Schematization present in Figure 2 represents the main phenomena: respect to the previous case (nonaggregative drug) the main difference is that here also the role of aggregation should be taken into account. In particular hydrogel network sequestrate drug monomers (adsorbing into the pores) that are not still available for aggregation. Consequently, in gel matrices dimers and trimers concentrations are minor than in water.

The drug total concentration is equal to:

$$
C_{\text {tot }}=C_{M}+2 C_{D}+\frac{1-\varepsilon}{\varepsilon} \frac{q^{\infty} \cdot K \cdot C_{M}}{1+K \cdot C_{M}}
$$

where $C_{M}$ is monomer concentration, $C_{D}$ is dimer concentration and $C_{t o t}$ the total drug present, $D_{M}$ is monomer diffusivity, $D_{\mathrm{D}}$ is dimer diffusivity.

Consequently, Equation (2) can be rewritten as:

$$
D_{\text {gel }}=\frac{\varepsilon}{\left(\varepsilon+(1-\varepsilon) \cdot \frac{q^{\infty} \cdot K}{\Delta^{2}}\right)} \cdot\left(\frac{C_{M}}{C_{\text {tot }}} \cdot D_{M}+\frac{C_{D}}{C_{\text {tot }}} \cdot D_{D}\right)
$$

Here the diffusivity of drug molecules in gel environment depends on points 1-5 described above and also on the tendency of drug molecules have to aggregate (black arrows in Figure 2) or not. 


\section{RESULTS AND DISCUSSION}

The wide spectrum of published experimental data addressed in this study is the following:

- 2-Hydroxyethyl methacrylate/methacrylic acid (HEMA/MAA) copolymer hydrogels with varying HEMA:MAA ratios (100:0, 99:1, 70:30, and 0:100) loaded with theophylline, acetazolamide, sodium fluorescein and riboflavin ${ }^{27}$ (fig. 3).

- Poly-hydroxyethyl methacrylate/poly-vinylpyrrolidone hydrogel (98:2), HEMA/PVP loaded with chlorhexidine, levofloxacin and diclofenac ${ }^{35}$ (fig. 4).

- 3-Tris(trimethylsilyloxy)silylpropyl-2-methylprop-2-enoate/N-vinylpyrrolidone/2-hydroxyethyl methacrylate (40:40:20), TRIS/NVP/ HEMA loaded with chlorhexidine, levofloxacin and diclofenac ${ }^{35}$ (fig. 5).

- Polymer 47K (Protein Polymer Technologies, San Diego, CA): a silkelastin like protein-based (SELP) block copolymer with an amino acid sequence motifs loaded with theophylline and vitamin $B 12^{33}$ (figs. 6 and 7).

In the first case examined, ${ }^{27}$ the authors investigated the molecular diffusion coefficients of four prototypical drugs in soft-contact lens material hydrogels of varying copolymer composition and aqueous $\mathrm{pH}$ using two-photon fluorescence confocal microscopy and UV/Vis-
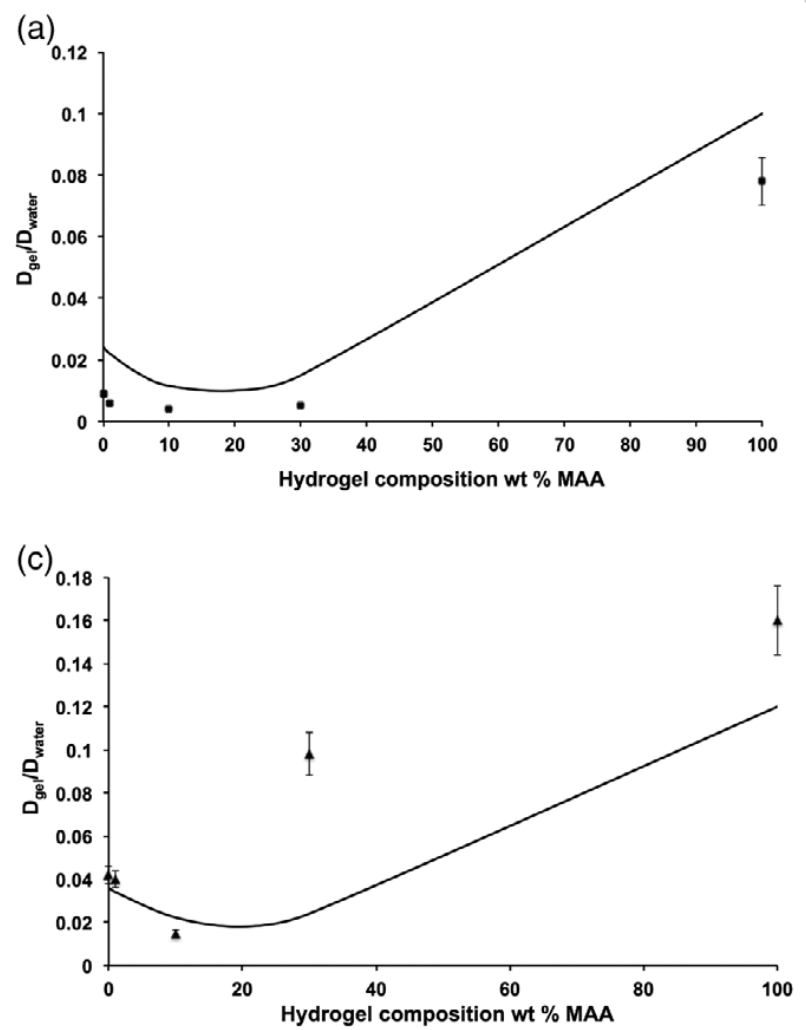

absorption spectrophotometry. The four molecules (drugs and drug 54 mimetics) tested were: riboflavin, sodium fluorescein, acetazolamide, 55 and theophylline. The hydrogels studied by Liu and coworkers were 56 based on copolymers of HEMA and MAA. To study the contribution of 57 the extent of the solute adsorption, the hydrogel copolymer composi- 58 tion was varied in HEMA:MAA weight ratios of 100:0, 99:1, 90:10, 59 70:30, and 0:100. Here, the authors considered the key role of adsorp- 60 tion, described by Langmuir isotherm and aggregation. Indeed, at the 6 concentration studied, all four drugs tend to form dimers. ${ }^{38-41}$

The mathematical model applied and described in the previous 63 section considers the following hypotheses: (a) drug molecules 64 adsorbed onto the three-dimensional hydrogel network in the mono- 65 meric state. The adsorption step indeed reduces the contribution of 66 any drug-aggregation phenomenon. As a consequence, at a low drug 67 concentration, the most important phenomenon is adsorption within 68 hydrogel pores, which reduces the amount of drug available for the 69 formation of dimers; (b) as the amount of the drug is increased, the 70 adsorption sites are then saturated and the drug can diffuse quicker, 71 as in water; the diffusion is driven only by the concentration gradient. 72 The rationale for this is based on the observation that the ratio 73 between the mean gel-network mesh size and the mean drug hydro- 74 dynamic radius is extremely low-diffusant. Molecules are mobile 75 inside the entangled hydrogel network, and thus, diffuse with a high 76 free motion. Therefore, the adsorption mechanism is expected to play 77 a dominant role at a low drug concentration, whereas its role is negli- 78 gible for a higher drug concentration. Equation (4) was therefore used 79

(b)

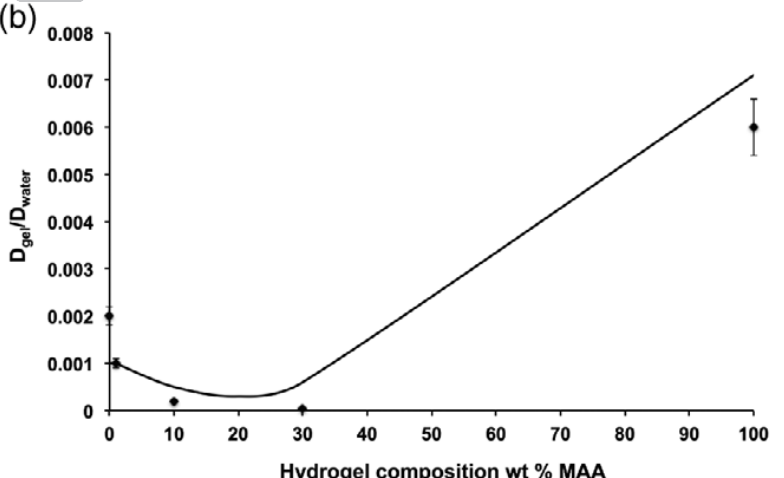

(d)

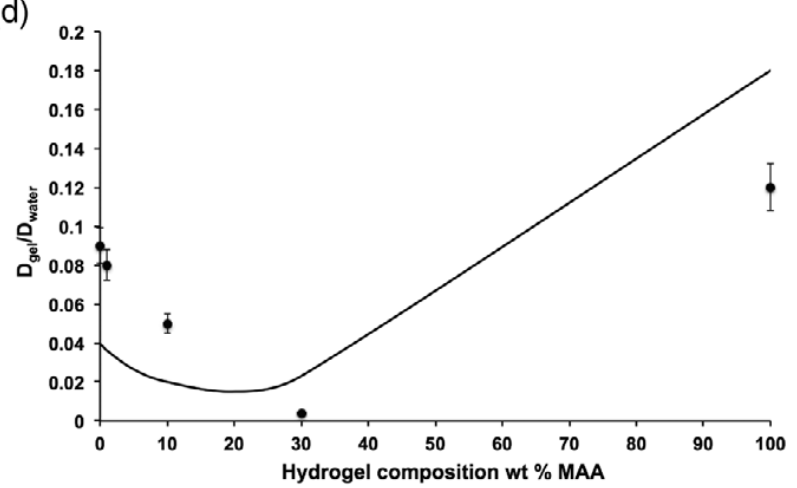
55 6 57 8 59 6 1 62 3 4 6 7 8 6 0

$$
2
$$
3 4 5 7 79

FIGURE 3 Comparison between the mathematical model (line) and the experimental results (dots) on drugs diffusivity from HEMA/MAA hydrogels: (a) riboflavin, (b) sodium fluorescein, (c) acetazolamide, and (d) theophylline. Experimental data obtained from Reference 27 
1 to model the ratio between drug diffusivity in gel and drug diffusivity in water $\left(D_{\text {gel }} / D_{\text {water }}\right)$ depending on the composition of the hydrogels (percentage of MAA). The comparison between the model trend and experimental values obtained is visible in Figure 3. $D_{\text {gel }} / D_{\text {water }}$ ratio initially declines with addition of MAA (0-10\%), then after a further addition of MAA (10-100\%) $D_{\text {gel }} / D_{\text {water }}$ rises for all solutes. Despite similar solute sizes, relative diffusion coefficients vary by orders of magnitude in HEMA-containing hydrogels of identical water content. This observation is again ascribed to reduced diffusion rates arising from specific interactions with HEMA-copolymer chains. Here, however, relative diffusion coefficients also vary by orders of magnitude in $100 \%$ MAA hydrogels, suggesting solute-specific interactions with electrically neutral MAA-copolymer strands described by different adsorption mechanisms. A greater reduction of $D_{\text {gel }} / D_{\text {water }}$ is expected to be exhibited by solutes of stronger specific interactions with MAA-copolymer. The model well matches quantitatively the observed experimental trends (Figure 3 ).

Pimenta and coworkers ${ }^{35}$ measured the equilibrium partitioning and the diffusion coefficients of several ophthalmic drugs, namely, chlorhexidine, levofloxacin, and diclofenac in two contact lens materials: a pHEMA based hydrogel (HEMA/PVP) and a silicone based hydrogel (TRIS/NVP/HEMA). The diffusion coefficients were experimentally determined from the drug release profiles, from samples

(a)

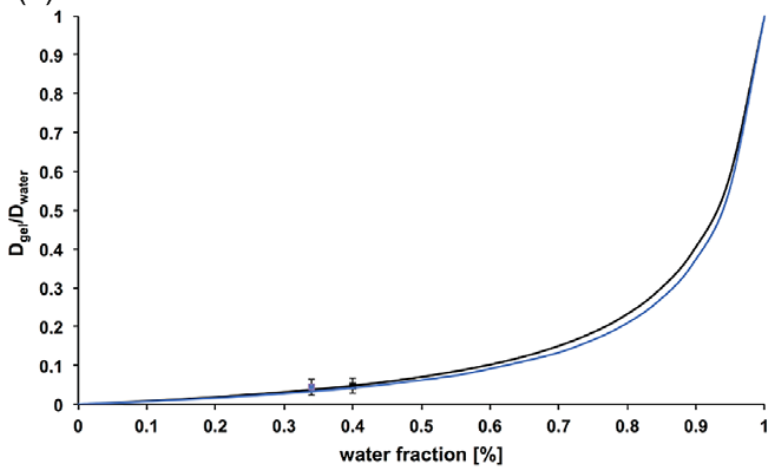

(C)

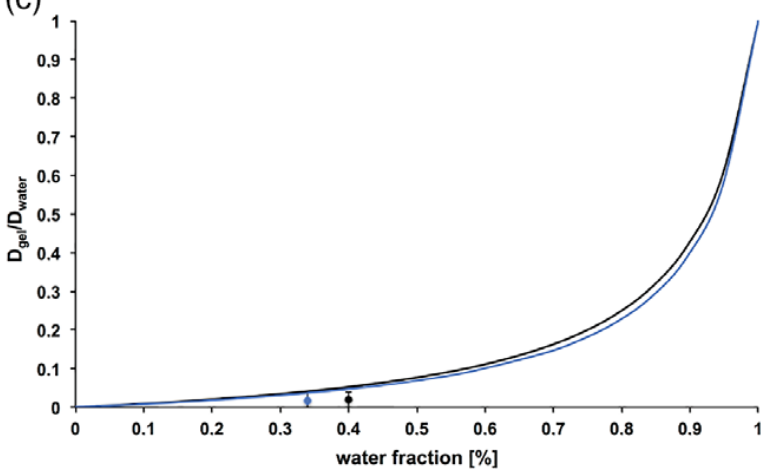

FIGURE 4 Comparison between the mathematical model (line) and the experimental results (dots) on drugs diffusivity from HEMA/PVP hydrogels (black) and TRIS/NVP/HEMA hydrogels (blue): (a) chlorhexidine, (b) levofloxacin, and (c) diclofenac. Experimental data obtained from Reference 35 [Color figure can be viewed at wileyonlinelibrary.com] loaded in sink conditions, and as expected depend on their hydrody- 54 namic radius. Here, the authors considered the key role of drugpolymer interaction (adsorption, by Langmuir isotherm) and drug-drug interactions (aggregation). Indeed, at the concentration studied, all three drugs tend to form dimers. ${ }^{42-44}$

Therefore, we used Equation (4) to model the ratio between drug diffusivity in gel and drug diffusivity in water $\left(D_{\text {gel }} / D_{\text {water }}\right)$. The comparison between the model trend and the experimental values obtained is visible in Figure 4. As expected, $D_{\text {gel }} / D_{\text {water }}$ ratio increases exponentially with the water fraction. Moreover, it is visibly clear that the model adequately reproduces the experimental trend and thus provides a good description of the synergic effects of both drugpolymer and drug-drug interactions.

Dinerman and coworkers ${ }^{33}$ studied the role of the molecular size and volume fraction on drug diffusivity through silk-based hydrogels. They highlighted that the Fickian diffusion represented the main phenomenon that took place in the two-hydrogel families they studied (silk-elastin like protein polymer hydrogel 1.1 and silk-elastin like protein polymer hydrogel 3.2 and 4.2).

Here, the authors added the influence of drug-polymer interactions since no information was found on dimer formation. The results of the model (line) obtained with Equation (2) compared with the experimental results (points) and good matches between them are visible in Figure 5.

(b)

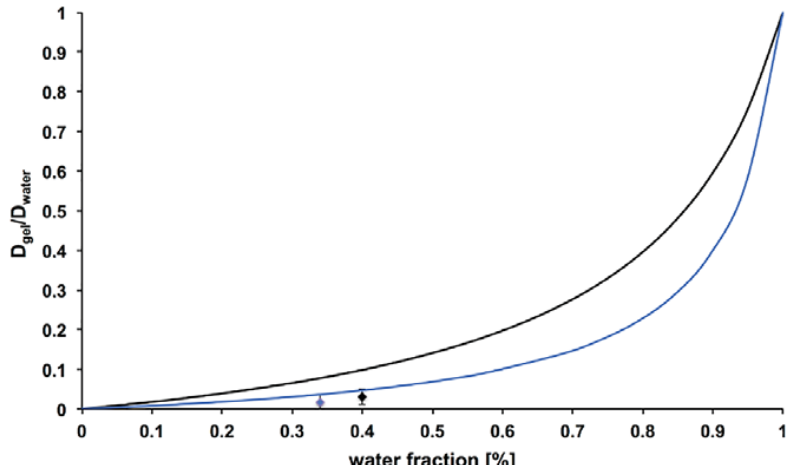



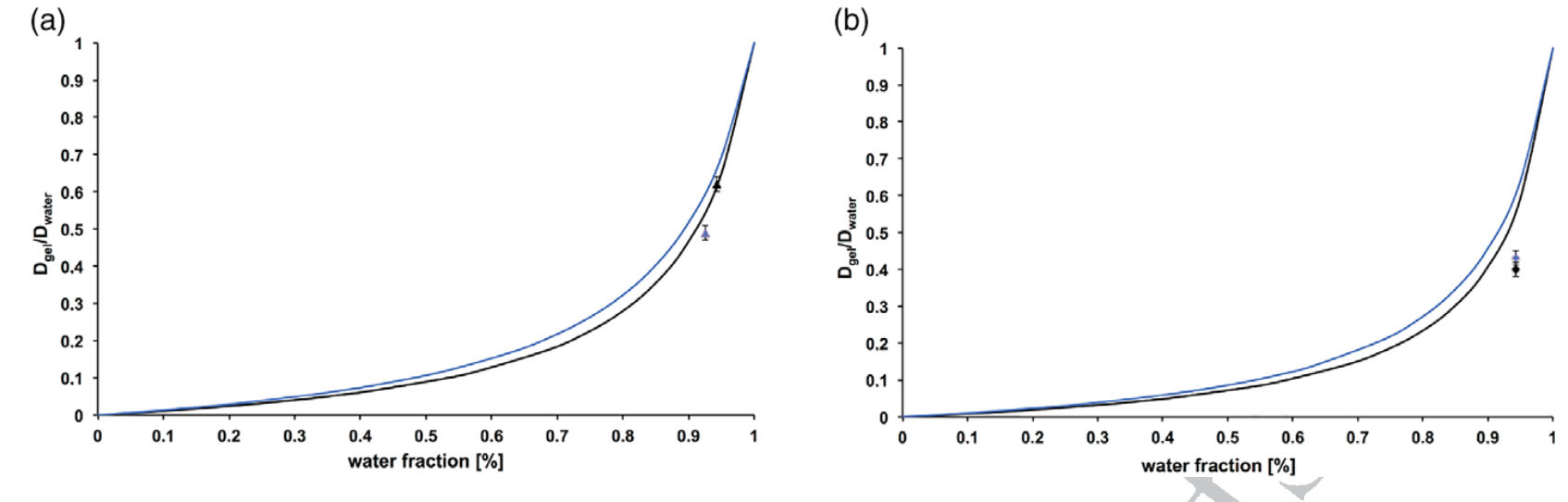

FIGURE 5 Comparison between the mathematical model (line) and the experimental results (dots) on drugs diffusivity from silk-elastin like protein polymer hydrogel 1.1 (black) and hydrogel 3.2 (blue): (a) theophylline and (b) vitamin B12. Experimental data obtained from Reference 33 [Color figure can be viewed at wileyonlinelibrary.com]

\section{4 | CONCLUSIONS}

To assess the predictive capability and model reliability of a previously derived mathematical model, and thus the validity of the needed hypothesis to formulate the equations starting from a chromatographic mass balance, this approach was validated against various and diverse sets of experimental data taken from literature. In particular, two mechanisms are very important and should be taken into account: the interaction between the mobile and the stationary phase (drugpolymer interactions) and between molecules of the mobile phase (drug-drug interactions). In all the cases examined, the simulation results exhibited a satisfactory quantitative match with the experimental data: this confirms the consistency of the hypothesis and the reliability of the chosen approach. A better match would be possible if adsorption parameters calculated experimentally could be used. These findings suggest that the model is capable of providing reasonable a priori predictions of the diffusion coefficient of a solute within hydrogel-based systems. Moreover, thanks to its simplicity and to the very low system requirements and CPU time-particularly with regard to FEM simulations-the authors' model allows to obtain immediate views of the system's behavior.

\section{ACKNOWLEDGMENTS}

Authors would like to thank Daniele Micale and Marco Cernigliaro for their help in literature search. Moreover, we would like to thank Prof. Mele and Prof. Morbidelli for fruitful discussions.

\section{NOTATION}

$C_{G} \quad$ drug concentration in gel, $\mathrm{mg} / \mathrm{mL}$

$C_{M} \quad$ monomer concentration, $\mathrm{mg} / \mathrm{mL}$

$C_{D} \quad$ dimer concentration, $\mathrm{mg} / \mathrm{mL}$

$D_{\text {gel }} \quad$ drug diffusivity in hydrogel, $\mathrm{m}^{2} / \mathrm{s}$

$D_{M} \quad$ monomer diffusivity in water, $\mathrm{m}^{2} / \mathrm{s}$

$D_{\mathrm{D}} \quad$ dimer diffusivity in water, $\mathrm{m}^{2} / \mathrm{s}$

$D_{\text {water }}$ drug diffusivity in water, $\mathrm{m}^{2} / \mathrm{s}$
$K \quad$ Langmuir isotherm parameter

$q$ adsorbed concentration, $\mathrm{mg} / \mathrm{cm}^{3}$

$q^{\infty} \quad$ maximum adsorbed concentration, $\mathrm{mg} / \mathrm{cm}^{3}$

\section{GREEK LETTERS}

$\varepsilon \quad$ porosity

ORCID

Filippo Rossi (1D) https://orcid.org/0000-0003-2665-120X

\section{REFERENCES}

1. Park K. Controlled drug delivery systems: past forward and future back. J Control Release. 2014;190:3-8.

2. Ulbrich K, Hola K, Subr V, Bakandritsos A, Tucek J, Zboril R. Targeted drug delivery with polymers and magnetic nanoparticles: covalent and noncovalent approaches, release control, and clinical studies. Chem Rev. 2016;116(9):5338-5431.

3. Fan JL, Wang SZ, Sun W, et al. Anticancer drug delivery systems based on inorganic nanocarriers with fluorescent tracers. AlChE J. 2018;64(3):835-859.

4. Deng C, Jiang YJ, Cheng R, Meng FH, Zhong ZY. Biodegradable polymeric micelles for targeted and controlled anticancer drug delivery: promises, progress and prospects. Nano Today. 2012;7(5):467-480.

5. Mauri E, Papa S, Masi M, Veglianese P, Rossi F. Novel functionalization strategies to improve drug delivery from polymers. Expert Opin Drug Deliv. 2017;14(11):1305-1313.

6. Choi M, Chung JH, Cho Y, Hong BY, Hong J. Nano-film modification of collagen hydrogels for controlled growth factor release. Chem Eng Sci. 2015;137(12):626-630.

7. Rossi F, Perale G, Papa S, Forloni G, Veglianese P. Current options for drug delivery to the spinal cord. Expert Opin Drug Deliv. 2013;10(3): 385-396.

8. Tandon B, Magaz A, Balint R, Blaker JJ, Cartmell SH. Electroactive biomaterials: vehicles for controlled delivery of therapeutic agents for drug delivery and tissue regeneration. J Control Release. 2018;129:148-168.

9. Caldorera-Moore ME, Liechty WB, Peppas NA. Responsive theranostic systems: integration of diagnostic imaging agents and responsive controlled release drug delivery carriers. Acc Chem Res. 2011:44(10):1061-1070. 
10. Chien JY, Ho RJY. Drug delivery trends in clinical trials and translational medicine: evaluation of pharmacokinetic properties in special populations. J Pharm Sci. 2011;100(1):53-58

11. Li JY, Mooney DJ. Designing hydrogels for controlled drug delivery. Nat Rev Mater. 2016;1(12):16071.

12. Upadhyay A, Kandi R, Rao CP. Injectable, self-healing, and stress sustainable hydrogel of BSA as a functional biocompatible material for controlled drug delivery in cancer cells. ACS Sustain Chem Eng. 2018;6 (3):3321-3330

13. Brown TE, Anseth KS. Spatiotemporal hydrogel biomaterials for regenerative medicine. Chem Soc Rev. 2017;46:6532-6552.

14. Liu W, Chen XD, Mercadè-Prieto R. Spatial quantification of hydrogels swelling using wide-field fluorescence microscopy. Chem Eng Sci. 2017;158(2):349-358.

15. Guo XH, Wang J, Li L, et al. Tunable polymeric hydrogels assembled by competitive complexation between cyclodextrin dimers and adamantyl substituted poly(acrylate)s. AIChE J. 2010;56(11):3021-3024.

16. Rossi F, Ferrari R, Papa S, et al. Tunable hydrogel-nanoparticles release system for sustained combination therapies in the spinal cord. Colloids Surf B. 2013;108:169-177.

17. Lai WF, He ZD. Design and fabrication of hydrogel-based nanoparticulate systems for in vivo drug delivery. I Control Release. 2016;243:269-282.

18. Azagarsamy MA, Marozas IA, Spans S, Anseth KS. Photoregulated hydrazone-based hydrogel formation for biochemically patterning 3D cellular microenvironments. ACS Macro Lett. 2016;5(1):24-28.

19. Perkins EL, Lowe JP, Edler KJ, Rigby SP. Studies of structuretransport relationships in biodegradable polymer microspheres for drug delivery using NMR cryodiffusometry. Chem Eng Sci. 2010;65(2): 611-625.

20. Russell SM, Belcher EB, Carta G. Protein partitioning and transport in supported cationic acrylamide-based hydrogels. AIChE J. 2005;49(5): 1168-1177.

21. Siepmann J, Siepmann F. Modeling of diffusion controlled drug delivery. J Control Release. 2012;161(2):351-362.

22. Peppas NA, Narasimhan B. Mathematical models in drug delivery: how modeling has shaped the way we design new drug delivery systems. J Control Release. 2014;190:75-81.

23. Lin CC, Metters AT. Hydrogels in controlled release formulations: network design and mathematical modeling. Adv Drug Deliv Rev. 2006;58 (12-13):1379-1408.

24. Amsden B. Diffusion in polyelectrolyte hydrogels: application of an obstruction-scaling model to solute diffusion in calcium alginate. Macromolecules. 2001;34(5):1430-1435.

25. Seiffert S, Sprakel J. Physical chemistry of supramolecular polymer networks. Chem Soc Rev. 2012;41(2):909-930.

26. Hadjiev NA, Amsden B. An assessment of the ability of the obstructionscaling model to estimate solute diffusion coefficients in hydrogels. J Control Release. 2015;199:10-16.

27. Liu DE, Dursch TJ, Taylor NO, Chan SY, Bregante DT, Radke CJ. Diffusion of water-soluble sorptive drugs in HEMA/MAA hydrogels. J Control Release. 2016;239:242-248.

28. Kotsmar C, Sells T, Taylor N, Liu DE, Prausnitz JM, Radke CJ. Aqueous solute partitioning and mesh size in HEMA/MAA hydrogels. Macromolecules. 2012:45(22):9177-9187.
29. Schirmer EB, Carta G. Protein adsorption in charged agarose gels studied by light microscopy. AIChE J. 2007;53(6):1472-1482.

30. Rossi F, Castiglione F, Salvalaglio M, et al. On the parallelism between the mechanisms behind chromatography and drug delivery: the role of interactions with a stationary phase. Phys Chem Chem Phys. 2017; 19:11518-11528.

31. Crank GS. Diffusion in polymers. New York; 1968.

32. Caccavo D, Cascone S, Lamberti G, Barba AA. Modeling the drug release from hydrogel-based matrices. Mol Pharm. 2015;12:474-483.

33. Dinerman AA, Cappello J, Ghandehari H, Hoag SW. Solute diffusion in genetically engineered silk-elastin like protein polymer hydrogels. J Control Release. 2002;82:277-287.

34. Dursch TJ, Taylor N, Liu DE, Wu RY, Prausnitz JM, Radke CJ. Watersoluble drug partitioning and adsorption in HEMA/MAA hydrogels. Biomaterials. 2014;35:620-629.

35. Pimenta AFR, Ascenso J, Fernandes JCS, Colaco R, Serro AP, Saramago B. Controlled drug release from hydrogels for contact lenses: drug partitioning and diffusion. Int J Pharm. 2016;515:467-475.

36. Rossi F, Castiglione F, Ferro M, et al. Drug-polymer interactions in hydrogel-based drug delivery systems: experimental and theoretical study. ChemPhysChem. 2015;16(13):2818-2825.

37. Rossi F, Castiglione F, Ferro M, Moioli M, Mele A, Masi M. The role of drug-drug interactions in hydrogel delivery systems: experimental and model study. ChemPhysChem. 2016;17(11):1615-1622.

38. Lopez Arbeloa I. Dimeric and trimeric states of the fluorescein dianion. J Chem Soc Faraday Trans. 1981;77:1735-1742.

39. Khamar D, Pritchard RG, Bradshaw IJ, Hutcheon GA, Seton L. Polymorphs of anhydrous theo-phylline: stable form IV consists of dimer pairs and metastable form I consists of hydrogen-bonded chains. Acta Crystallogr C. 2011;C67:0496-0499.

40. Baranovskii SF, Bolotin PA. Association of riboflavin, caffeine, and sodium salicylate in aqueous solution. J Appl Spectrosc. 2007;74(2): 211-218.

41. Ozel AE, Gunduz SK, Celik S, Akyuz S. Structural and vibrational study on monomer and dimer forms and water clusters of acetazolamide. J Spectrosc. 2013;2013:538917.

42. Keen OS, Thurman EM, Ferrer I, Dotson AR, Linden KG. Dimer formation during UV photolysis of diclofenac. Chemosphere. 2013;93:19481956.

43. Shakeel M, Mehmood K, Siddiq M. Aggregation properties of levofloxacin in water and ethanol and its interaction with sodium dodecyl sulphate: a thermodynamic study. J Chem Sci. 2015;127(11):20732079.

44. Zeng P, Zhang G, Rao A, Bowles W, Wiedmann TS. Concentration dependent aggregation properties of chlorhexidine salts. Int J Pharm. 2009;367:73-78.

How to cite this article: Rossi F, Masi M. Ability of chromatographic mass balance to predict solute diffusivity in drug delivery systems. AIChE J. 2019;e16709. https://doi.org/ 10.1002/aic.16709 